\title{
An Integral Formula for the Maslov-Index of a Symplectic Path
}

\author{
Stefan Bechtluft-Sachs \\ Department of Mathematics, NUI Maynooth, Co Kildare, Ireland \\ e-mail: stefan@maths.nuim.ie
}

November 8, 2007

\begin{abstract}
The Maslov index of a not necessarily closed path $M$ in the symplectic group $\operatorname{Sp}(2 n)$ is expressed by an integral formula. We have an explicit formula for the integrand which is a rational 1-form on $\operatorname{Sp}(2 n)$.
\end{abstract}

Keywords: Symplectic Group, Lagrangian Grassmannian, Maslov Index

\section{Introduction}

The homotopy class of a loop $\Lambda(t)$ in the Lagrangian Grassmannian $\mathbf{L}(2 n)$ in standard symplectic space $\mathbb{R}^{2 n}$ is detected by an integer, the Maslov index. Following Arnold, [1], this can be defined as an intersection number with a certain subset of $\mathbf{L}(2 n)$, the Maslov cycle. In [6 this definition of the Maslov index was extended to not necessarily closed paths, and it is this index which enters in the Gutzwiller trace formula in semiclassical quantisation, 2, see also [4.

The group $\operatorname{Sp}(2 n)$ of symplectic automorphisms of $\mathbb{R}^{2 n}$ acts transitively on the set of Lagrangians and thus $\mathbf{L}(2 n)=\mathrm{Sp}(2 n) / \mathrm{H}(2 n)$ is a homogeneous space. In applications the path $\Lambda(t)$ is frequently given in the form $M(t) \Lambda_{0}$ with a curve $M(t) \in \operatorname{Sp}(2 n)$ of symplectic automorphisms and a fixed Lagrangian $\Lambda_{0}$. In view of calculations of the Maslov index as in 3 it seems desirable to compute the Maslov index $\mu(\Lambda)=\mu(M)$ directly in terms of $M$.

In the present note we derive such a formula. We show that the Maslov index is the line integral over a certain differential form $\chi_{\mathrm{Sp}(2 n)} \in \Omega^{1}(\operatorname{Sp}(2 n), \mathbb{R})$ plus end point terms which are also given by an explicit function $\Phi$ on $\operatorname{Sp}(2 n)$.

For unitary paths, i.e. paths $M(t) \in \mathrm{U}(n) \subset \mathrm{Sp}(2 n)$ in the unitary group the (complex) Trace $\operatorname{Tr}_{\mathbb{C}}$ on the Lie algebra $\mathfrak{u}(n)$ is an invariant for the adjoint representation, and therefore provides a bi-invariant 1-form on $\mathrm{U}(n)$. On the symplectic group $\operatorname{Sp}(2 n)$ however a nontrivial biinvariant 1-form does not exist. The 1-form $\chi_{\operatorname{Sp}(2 n)}$ and the function $\Phi$ we construct are invariant under the 
left action of $\operatorname{Sp}(2 n)$ and under the right action of the stabilizer subgroup $\mathrm{H}(n)$ which contains $\mathrm{O}(n)$.

We formulate our main result in the next section 2 . Then, in section 3 , we compute the 1-form $\chi_{\operatorname{Sp}(2 n)}$ explicitely in terms of the entries of the symplectic matrix. Finally we prove our integral formula by verifying the axioms for the Maslov index in section 4.

\section{A local formula for the Maslov index}

We need to introduce some notation and recall some well known facts from symplectic geometry as may be found in [5], for instance. Let $\Lambda_{0}$ be a Lagrangian in $\mathbb{R}^{2 n}$ and $\Lambda_{0}^{\perp}$ the orthogonal complement with respect to the standard scalar product $\langle\cdot \mid \cdot\rangle$. The symplectic structure $\omega$ on $\mathbb{R}^{2 n}$ can be written as

$$
\omega(x, y)=\langle x \mid J y\rangle
$$

where $J$ is a complex structure, i.e. $J \in \mathrm{O}(2 n)$ with $J^{2}=-\mathbf{1}_{2 n}$ and we have $J \Lambda_{0}=\Lambda_{0}^{\perp}$.

Usually we choose coordinates such that $\Lambda_{0}=\mathbb{R}^{n} \times\{0\}, \Lambda_{0}^{\perp}=\{0\} \times \mathbb{R}^{n}$ and so that the complex structure $J$ becomes

$$
J=\left(\begin{array}{cc}
0 & -\mathbf{1}_{n} \\
\mathbf{1}_{n} & 0
\end{array}\right) \quad, \quad \mathbf{1}=\left(\begin{array}{ccc}
1 & 0 & 0 \\
0 & \ddots & 0 \\
0 & 0 & 1
\end{array}\right) \in \operatorname{Mat}(n \times n, \mathbb{R})
$$

With respect to the orthogonal decomposition

$$
\mathbb{R}^{2 n}=\Lambda_{0} \oplus \Lambda_{0}^{\perp}
$$

we can write a (symplectic) matrix $M$ as

$$
M=\left(\begin{array}{ll}
a & b \\
c & d
\end{array}\right)
$$

where

$$
a \in \operatorname{Hom}\left(\Lambda_{0}, \Lambda_{0}\right), b \in \operatorname{Hom}\left(\Lambda_{0}^{\perp}, \Lambda_{0}\right), c \in \operatorname{Hom}\left(\Lambda_{0}, \Lambda_{0}^{\perp}\right), d \in \operatorname{Hom}\left(\Lambda_{0}^{\perp}, \Lambda_{0}^{\perp}\right) .
$$

All these will be identified with $\operatorname{Mat}(n \times n ; \mathbb{R})$.

By means of the complex structure (1) we can read a complex $(n \times n)$-matrix $Y$ as a real $(2 n \times 2 n)$-matrix $\left(\begin{array}{cc}\alpha & -\beta \\ \beta & \alpha\end{array}\right)$. The traces $\operatorname{Tr}_{\mathbb{C}}$ over $\mathbb{C}$ respectively $\operatorname{Tr}=\operatorname{Tr}_{\mathbb{R}}$ over $\mathbb{R}$ are related by

$$
\operatorname{Tr}_{\mathbb{C}}(Y)=\frac{1}{2} \operatorname{Tr}(Y)-\frac{i}{2} \operatorname{Tr}(J Y)=\operatorname{Tr}(\alpha)+i \operatorname{Tr}(\beta) .
$$


The group $\operatorname{Sp}(2 n)$ of symplectic automorphisms is

$$
\mathrm{Sp}(2 n)=\left\{M \in \mathrm{Gl}(2 n) \mid M^{t} J M=J\right\}
$$

and acts transitively on the Lagrangian Grassmannian $\mathbf{L}(2 n)$. The stabilizer group, i.e. the isotropy group of this action at $\Lambda_{0}=\mathbb{R}^{n} \times\{0\}$ is

$$
\mathrm{H}(n)=\left\{\left(\begin{array}{cc}
A & X \\
0 & \left(A^{t}\right)^{-1}
\end{array}\right) \mid A \in \operatorname{Gl}(n), A X \in \operatorname{Mat}(n \times n, \mathbb{R}) \text { symmetric }\right\}
$$

We will also need the subgroup $\mathrm{H}^{+}(n)$ consisting of those matrices in $\mathrm{H}(n)$ with $A$ upper triangular and positive. The unitary group $\mathrm{U}(n)$ and the orthogonal group $\mathrm{O}(n)$ are naturally identified as subgroups of $\operatorname{Sp}(2 n)$,

$$
\begin{aligned}
\mathrm{U}(n) & =\{M \in \mathrm{Sp}(2 n) \mid M J=J M\}=\mathrm{Sp}(2 n) \cap \mathrm{O}(2 n) \\
\mathrm{O}(n) & =\left\{M \in \mathrm{Sp}(2 n) \mid M J=J M \text { and } M \Lambda_{0}=\Lambda_{0}\right\} \\
& =\left\{\left(\begin{array}{cc}
T & 0 \\
0 & T
\end{array}\right) \mid T \in \mathrm{O}(n)\right\}=\mathrm{H}(n) \cap \mathrm{O}(2 n) \subset \mathrm{U}(n) .
\end{aligned}
$$

With these identifications we can represent the Lagrangian Grassmannian as homogeneous space,

$$
\mathbf{L}(2 n)=\mathrm{Sp}(2 n) / \mathrm{H}(n)=\mathrm{U}(n) / \mathrm{O}(n) .
$$

The square of the (complex) determinant

$$
\mathbf{L}(2 n)=\mathrm{U}(n) / \mathrm{O}(n) \rightarrow \mathrm{U}(1), F \mapsto \operatorname{det}_{\mathbb{C}}(F)^{2}
$$

induces an isomorphism of the fundamental groups

$$
\pi_{1}(\mathbf{L}(2 n)) \cong \pi_{1}(\mathrm{U}(1))=\mathbb{Z} .
$$

The Jordan-, or QR- decomposition, of the symplectic group is

$$
\mathrm{Sp}(2 n)=\mathrm{U}(n) \cdot \mathrm{H}^{+}(n)
$$

and

$$
\mathrm{H}^{+}(n)=\mathrm{D}^{+}(n) \ltimes \operatorname{Sym}(n)
$$

is the semidirect product of the group of positive upper triangular matrices with the vector group of symmetric matrices. In particular, we have a diffeomorphism and a homotopy equivalence, both equivariant under the right action of $\mathrm{H}^{+}(n)$,

$$
\mathcal{F}: \operatorname{Sp}(2 n) \cong \mathrm{U}(n) \times \mathrm{H}^{+}(n) \stackrel{\simeq}{\longrightarrow} \mathrm{U}(n) .
$$

We will also need the map

$$
\mathcal{H}: \operatorname{Sp}(2 n) \cong \mathrm{U}(n) \times \mathrm{H}^{+}(n) \stackrel{\simeq}{\longrightarrow} \mathrm{H}^{+}(n) .
$$


These are defined by first decomposing a given $M \in \operatorname{Sp}(2 n), M=F H$ with $F \in \mathrm{U}(n), H \in \mathrm{H}^{+}(n)$ uniquely determined by $M$. This can be done efficiently by any of the algorithms for the QR-decomposition. Then we set $\mathcal{F}(M):=F$ and $\mathcal{H}(M):=H$

For the contribution of end points we need a function

$$
\Phi: \operatorname{Sp}(2 n) \rightarrow \mathbb{R} .
$$

which is defined as follows: $\Phi(M)$ is computed from the unitary part

$$
\mathcal{F}(M)=\left(\begin{array}{cc}
\alpha & -\beta \\
\beta & \alpha
\end{array}\right)
$$

alone. Since $F^{t} F=\mathbf{1}_{2 n}=F F^{t}$ we have

$$
\begin{aligned}
& \alpha^{t} \alpha+\beta^{t} \beta=\mathbf{1}_{n}, \alpha^{t} \beta=\beta^{t} \alpha, \\
& \alpha \alpha^{t}+\beta \beta^{t}=\mathbf{1}_{n}, \alpha \beta^{t}=\beta \alpha^{t} .
\end{aligned}
$$

From these relations it follows that $\alpha^{t} \alpha, \beta^{t} \beta$ and $\alpha^{t} \beta$ commute and can therefore be simultaneously diagonalized. Since $\operatorname{Spec}\left(\beta^{t} \beta\right) \subset[0,1]$ we may set

$$
X:=\arcsin \left(\left(\beta^{t} \beta\right)^{1 / 2}\right) \in \operatorname{Mat}(n \times n ; \mathbb{R}) .
$$

Note that $X$ is nonegative symmetric and $\operatorname{Spec} X \subset\left[0, \frac{\pi}{2}\right]$. Now define $T_{\alpha}, T_{\beta} \in$ $\operatorname{Mat}(n \times n ; \mathbb{R})$ as

$$
T_{\beta}=\beta \sin (X)^{-1} \operatorname{pr}_{(\operatorname{ker} X)^{\perp}}, T_{\alpha}=\alpha \cos (X)^{-1} \operatorname{pr}_{\operatorname{ker}\left(X-\frac{\pi}{2} \mathbf{1}_{n}\right)^{\perp}}
$$

where pr denotes the orthogonal projection on the subspace indicated. We then have

$$
\beta=T_{\beta} \sin (X), \alpha=T_{\alpha} \cos (X), T_{\beta}^{t} T_{\beta}=\operatorname{pr}_{(\operatorname{ker} X)^{\perp}}, T_{\alpha}^{t} T_{\alpha}=\operatorname{pr}_{\operatorname{ker}\left(X-\frac{\pi}{2} \mathbf{1}_{n}\right)^{\perp}}
$$

We define

$$
\Phi(M):=\frac{1}{\pi} \operatorname{Tr}\left(T_{\alpha}^{t} T_{\beta}\left(X-\frac{\pi}{2} \mathbf{1}_{n}\right)\right) .
$$

This is to arrange for $\mathbf{d} \Phi=\chi_{\mathrm{Sp}(2 n)}$ on the complement of the Maslov cycle (see Lemma 21 and (23) in the proof of that Lemma).

We can now write down an integral formula for the Maslov index:

Theorem Let $M:[0,1] \rightarrow \operatorname{Sp}(2 n)$ be a differentiable path in the symplectic group of $\mathbb{R}^{2 n}$ and let $\Lambda_{0} \subset \mathbb{R}^{2 n}$ be a fixed Lagrangian subspace. Then the Maslov index $\mu(\Lambda) \in \frac{1}{2} \mathbb{Z}$ of the path $\Lambda:[0,1] \rightarrow \mathbf{L}(2 n), \Lambda(t)=M(t) \Lambda_{0}$, in the Lagrangian-Grassmannian of $\mathbb{R}^{2 n}$ is given by

$$
\mu(M)=\mu(\Lambda)=\int_{M} \chi_{\mathrm{Sp}(2 n)}-\Phi(M(1))+\Phi(M(0))
$$


where $\Phi$ is as defined in 13 and

$$
\chi_{\mathrm{Sp}(2 n)}=-\frac{1}{2 \pi} \operatorname{Tr}\left(J M^{t} \mathbf{d} M\right)-\operatorname{Tr}\left(J \mathcal{H}(M)^{t} \mathbf{d}(\mathcal{H}(M))\right)
$$

This form is explicitely computed in (17) in terms of the coefficients of $M$.

Recall that the stabilizer part $H=\mathcal{H}(M) \in \mathrm{H}^{+}(n) \subset \operatorname{Mat}(2 n \times 2 n)$ is uniquely determined from $M$ by the following conditions (see $(9)$ ):

1. $\left.M^{t} M=H^{t} H\right)$,

2. $H=\left(\begin{array}{cc}A & A X \\ 0 & \left(A^{t}\right)^{-1}\end{array}\right)$ w.r.t. the splitting $\mathbb{R}^{2 n}=\Lambda_{0} \oplus \Lambda_{0}^{\perp}$,

3. $A \in \operatorname{Mat}(n \times n, \mathbb{R})$ is upper triangular with positive eigenvalues,

4. $X \in \operatorname{Mat}(n \times n, \mathbb{R})$ is symmetric.

\section{The 1-form on the symplectic group}

We will now derive explicit formulas for the integrand in (14). The form $\chi=$ $\frac{1}{2 \pi i} z^{-1} \mathbf{d} z$ on $U(1)=S^{1} \subset \mathbb{C}$ detects the homotopy type of a loop in $\mathrm{U}(1)$. To find the right 1 -form for the Maslov index we first pull back $\chi$ to $\mathrm{U}(n)$ via the map $\operatorname{det}_{\mathbb{C}}^{2}$ in (7). Sine $\mathbf{d}\left(\operatorname{det}_{\mathbb{C}} F\right)=\operatorname{det}_{\mathbb{C}}(F) \operatorname{Tr}_{\mathbb{C}}\left(F^{-1} \mathbf{d} F\right)$ we get

$$
\chi_{\mathrm{U}(n)}=\left(\operatorname{det}_{\mathbb{C}}^{2}\right)^{*} \chi=\frac{1}{\pi i} \operatorname{Tr}_{\mathbb{C}} F^{-1} \mathbf{d} F \in \Omega^{1}(\mathrm{U}(n)) .
$$

This already gives a formula, similiar to that for the winding number, for the Maslov index of a unitary path $F(t) \Lambda_{0}, F:[0,1] \rightarrow \mathrm{U}(n)$,

$$
\mu(F)=\frac{1}{\pi i} \int \operatorname{Tr}_{\mathbb{C}}\left(F^{-1} \mathbf{d} F\right)-\Phi(F(1))+\Phi(F(0))
$$

The 1-form on $\operatorname{Sp}(2 n)$ we are looking for is the pull back of $\chi_{\mathrm{U}(n)}$ over the map $\mathcal{F}$ in 8 . We compute this explicitely.

Lemma 15 Denote by $a, b, c, d \in \operatorname{Mat}(n \times n, \mathbb{R})$ the components of a symplectic matrix $M$, i.e the matrices such that

$$
M=\left(\begin{array}{ll}
a & b \\
c & d
\end{array}\right)
$$

with respect to the decomposition (2). Then

$$
\begin{aligned}
\chi_{\mathrm{Sp}(2 n)}=F^{*} \chi_{\mathrm{U}(n)}= & \frac{1}{\pi i} \operatorname{Tr}_{\mathbb{C}}\left(\mathcal{F}(M)^{-1} \mathbf{d} \mathcal{F}(M)\right) \\
= & \frac{-1}{2 \pi} \operatorname{Tr}\left(J M^{t} \mathbf{d} M-J H^{t} \mathbf{d} H\right) \\
= & \frac{-1}{2 \pi} \operatorname{Tr}\left[-b^{t} \mathbf{d} a-d^{t} \mathbf{d} c+a^{t} \mathbf{d} b+c^{t} \mathbf{d} d\right. \\
& \left.\quad-\left(a^{t} a+c^{t} c\right) \mathbf{d}\left[\left(a^{t} a+c^{t} c\right)^{-1}\left(a^{t} b+c^{t} d\right)\right]\right]
\end{aligned}
$$


Proof: $\quad$ Let $M \in \mathrm{Sp}(2 n)$ and $F=\mathcal{F}(M)=M H^{-1} \in \mathrm{U}(n)$ with $H \in \mathrm{H}^{+}(n)$. We get

$$
F^{-1} \mathbf{d} F=H M^{-1}(\mathbf{d} M) H^{-1}+H M^{-1} M \mathbf{d}\left(H^{-1}\right) \in \Omega^{1}(\operatorname{Sp}(2 n), \mathfrak{u}(n)) .
$$

Because of (4) and since $H^{t} H=M^{t} M$,

$$
\begin{aligned}
\operatorname{Tr}_{\mathbb{C}} F^{-1} \mathbf{d} F & =\frac{1}{2 i} \operatorname{Tr} J F^{-1} d F=\frac{1}{2 i} \operatorname{Tr}\left(J H M^{-1}(\mathbf{d} M) H^{-1}+J H M^{-1} M \mathbf{d}\left(H^{-1}\right)\right) \\
& =\frac{1}{2 i} \operatorname{Tr}\left(H^{-1} J H M^{-1} \mathbf{d} M-J H H^{-1}(\mathbf{d} H) H^{-1}\right) \\
& =\frac{1}{2 i} \operatorname{Tr}\left(J H^{t} H M^{-1} \mathbf{d} M-J(\mathbf{d} H) H^{-1}\right) \\
& =\frac{1}{2 i} \operatorname{Tr}\left(J M^{t} M M^{-1} \mathbf{d} M+J(\mathbf{d} H) J H^{t} J\right) \\
& =\frac{1}{2 i} \operatorname{Tr}\left(J M^{t} \mathbf{d} M-J H^{t} \mathbf{d} H\right)
\end{aligned}
$$

In view of 16 we can simplify this further. First

$$
\begin{aligned}
\operatorname{Tr}\left(J M^{t} \mathbf{d} M\right) & =\operatorname{Tr}\left(\begin{array}{cc}
0 & -\mathbf{1} \\
\mathbf{1} & 0
\end{array}\right)\left(\begin{array}{cc}
a^{t} & c^{t} \\
b^{t} & d^{t}
\end{array}\right)\left(\begin{array}{cc}
\mathbf{d} a & \mathbf{d} b \\
\mathbf{d} c & \mathbf{d} d
\end{array}\right) \\
& =\operatorname{Tr}\left(-b^{t} \mathbf{d} a-d^{t} \mathbf{d} c+a^{t} \mathbf{d} b+c^{t} \mathbf{d} d\right)
\end{aligned}
$$

For the second summand in 18 let $H=\left(\begin{array}{cc}A & A X \\ 0 & \left(A^{t}\right)^{-1}\end{array}\right) \in \mathrm{H}^{+}(n)$. We compute

$$
\begin{aligned}
\operatorname{Tr}\left(J H^{t} \mathbf{d} H\right) & =\operatorname{Tr}\left(\begin{array}{cc}
0 & -\mathbf{1} \\
\mathbf{1} & 0
\end{array}\right)\left(\begin{array}{cc}
A^{t} & 0 \\
X^{t} A^{t} & A^{-1}
\end{array}\right)\left(\begin{array}{cc}
\mathbf{d} A & \mathbf{d}(A X) \\
0 & \mathbf{d}\left(\left(A^{t}\right)^{-1}\right)
\end{array}\right) \\
& =\operatorname{Tr}\left(-X^{t} A^{t} \mathbf{d} A+A^{t} \mathbf{d}(A X)\right) \\
& =\operatorname{Tr}\left(A^{t} A \mathbf{d} X\right)
\end{aligned}
$$

since $X$ is symmetric. As $H^{t} H=M^{t} M$ we have $A^{t} A=a^{t} a+c^{t} c$ and $A^{t} A X=$ $a^{t} b+c^{t} d$ which yields

$$
\operatorname{Tr}\left(J H^{t} \mathbf{d} H\right)=\operatorname{Tr}\left[\left(a^{t} a+c^{t} c\right) \mathbf{d}\left[\left(a^{t} a+c^{t} c\right)^{-1}\left(a^{t} b+c^{t} d\right)\right]\right]
$$

\section{The axiomatic characterization of the Maslov index}

For the proof of the Theorem, denote by $\widetilde{\mu}(M)$ the right hand side of (14) for a path $M(t) \in \operatorname{Sp}(2 n)$. In order to show that $\widetilde{\mu}$ coincides with the Maslov 
index, we will check that $\widetilde{\mu}$ satisfies the five properties which were shown to characterize the Maslov index by Theorem 4.1 in [6].

We need two Lemmas. The first will permit us to move the path by paths in $\mathrm{O}(n)$.

Lemma 19 The form $\chi_{\mathrm{Sp}(2 n)} \in \Omega^{1}(\operatorname{Sp}(2 n))$ from (17) and the function $\Phi$ of (13) are left $-\mathrm{O}(n)$ and right $-\mathrm{H}(n)$-invariant. Thus if $M(t) \in \mathrm{Sp}(2 n)$ and $T(t) \in$ $\mathrm{O}(n), H(t) \in \mathrm{H}(n)$ are smooth paths, then

$$
\widetilde{\mu}(M)=\widetilde{\mu}(T M H) .
$$

Proof: Let

$M=F H^{+} \quad$ and $\quad H=T_{1} H_{1}^{+} \quad$ with $\quad F \in \mathrm{U}(n), H^{+}, H_{1}^{+} \in \mathrm{H}^{+}(n), T_{1} \in \mathrm{O}(n)$.

Then

$$
\mathcal{F}(T M H)=\mathcal{F}\left(T F H^{+} T_{1} H_{1}^{+}\right)=\mathcal{F}\left(T F T_{2} H_{2}^{+}\right)=T F T_{2}
$$

with some $T_{2}=\mathcal{F}\left(H^{+} T_{1} H_{1}^{+}\right) \in \mathrm{O}(n)$ and some $H_{2}^{+} \in \mathrm{H}^{+}(n)$, since

$$
\mathrm{H}(n)=\mathrm{O}(n) \cdot \mathrm{H}^{+}(n)=\mathrm{H}^{+}(n) \cdot \mathrm{O}(n) .
$$

Now

$$
\begin{aligned}
\operatorname{Tr}_{\mathbb{C}}\left(\left(T F T_{2}\right)^{-1} \mathbf{d}\left(T F T_{2}\right)\right)= & \operatorname{Tr}_{\mathbb{C}}\left(\left(T F T_{2}\right)^{-1} T F\left(\mathbf{d} T_{2}\right)\right) \\
& +\operatorname{Tr}_{\mathbb{C}}\left(\left(T F T_{2}\right)^{-1} T(\mathbf{d} F) T_{2}\right) \\
& +\operatorname{Tr}_{\mathbb{C}}\left(\left(T F T_{2}\right)^{-1}(\mathbf{d} T) F T_{2}\right) \\
= & \operatorname{Tr}_{\mathbb{C}}\left(F^{-1} \mathbf{d} F\right)
\end{aligned}
$$

since $T^{-1} \mathbf{d} T$ lies in the Lie algebra of $\mathrm{O}(n)$ and therefore $\operatorname{Tr}_{\mathbb{C}}\left(T^{-1} \mathbf{d} T\right)=0$.

For the invariance of the function $\Phi$ of $(13)$ it suffices to prove left and right invariance under $\mathrm{O}(n)$, because of $(20)$ as before. We also may assume $M=F$ unitary since right- $\mathrm{H}^{+}(n)$-invariance of $\Phi$ is clear from its definition. So let

$$
F=\left(\begin{array}{cc}
\alpha & -\beta \\
\beta & \alpha
\end{array}\right)
$$

be unitary with real $(n \times n)$-matrices $\alpha$ and $\beta$. We then have

$$
T F=\left(\begin{array}{cc}
T & 0 \\
0 & T
\end{array}\right)\left(\begin{array}{cc}
\alpha & -\beta \\
\beta & \alpha
\end{array}\right)=\left(\begin{array}{cc}
T \alpha & -T \beta \\
T \beta & T \alpha
\end{array}\right)
$$

From $(12)$ and 11 we see that this replaces the matrices $T_{\alpha}, T_{\beta}$ used to define $\Phi$ by $T T_{\alpha}, T T_{\beta}$ while leaving $X$ unchanged. By definition $(13) \Phi(T F)=\Phi(F)$. Similiarly, multiplying $T$ from the right, replaces $\alpha, \beta$ by $\alpha T, \beta T$. Thus, by 12 and (11), $T_{\alpha}, T_{\beta}, X$ are replaced by $T_{\alpha} T, T_{\beta} T$ and $T^{-1} X T$, since $\sin \left(T^{-1} X T\right)=$ $T^{-1} \sin (X) T$. By (13) and the conjugation invariance of the trace, $\Phi$ again is unchanged.

By the next Lemma $\widetilde{\mu}$ vanishes on a path $M(t)$ such that $M(t) \Lambda_{0}$ stays away from the Maslov cycle $\left\{\Lambda \in \mathbf{L}(2 n) \mid \Lambda \cap \Lambda_{0} \neq 0\right\}$. Thus, away from the Maslov cycle, the function $\Phi$ is an integral of $\chi_{\mathrm{Sp}(2 n)}$. 
Lemma 21 For $M \in \operatorname{Sp}(2 n)$ let $\beta(M), \alpha(M) \in \operatorname{Mat}(n \times n ; \mathbb{R})$ be the matrices in the unitary part

$$
\mathcal{F}(M)=\left(\begin{array}{cc}
\alpha(M) & -\beta(M) \\
\beta(M) & \alpha(M)
\end{array}\right)
$$

of $M$ as in (10). Then on the set $\operatorname{Sp}^{\times}(2 n)=\{M \mid \beta(M)$ invertible $\}$ we have $\mathbf{d} \Phi=\chi_{S p(2 n)}$.

In particular $\widetilde{\mu}$ vanishes on paths staying in $\mathrm{Sp}^{\times}(2 n)$.

The set $S p^{\times}(2 n)$ is the set of all $M$ such that $M(t) \Lambda_{0} \cap \Lambda_{0}=0$. Thus $S p^{\times}(2 n)$ is the preimage of the complement of the Maslov cycle under the projection $\operatorname{Sp}(2 n) \rightarrow \mathbf{L}(2 n)$.

Proof: It suffices to prove the Lemma on $\mathrm{U}^{\times}(n)=\mathrm{U}(n) \cap \mathrm{Sp}^{\times}(2 n)$ since both the form $\chi_{\mathrm{Sp}(2 n)}$ and $\Phi$ are right- $\mathrm{H}^{+}(n)$ invariant. We will now show that $\int_{F} \chi_{(2 n)}=\left[\Phi(F(t)]_{t=0}^{t=1}\right.$ for arbitrary paths $F:[0,1] \rightarrow \mathrm{U}(n)$.

Let $M=F \in \mathrm{U}^{\times}(n)$. Then the matrices $X(F)$ and $T_{\beta}(F)$ defined as in 11. and $(12)$ are invertible and depend smoothly on $F$. Let

$$
\begin{gathered}
X_{s}:=(1-s) \frac{\pi}{2} \mathbf{1}_{n}+s X(F) \quad \text { for } \quad s \in[0,1] \quad \text { and } \\
\alpha_{s}=T_{\alpha(F)} \cos \left(X_{s}\right) \quad, \quad \beta_{s}=T_{\beta(F)} \sin \left(X_{s}\right) .
\end{gathered}
$$

The path

$$
F_{s}=\left(\begin{array}{cc}
\alpha_{s} & -\beta_{s} \\
\beta_{s} & \alpha_{s}
\end{array}\right)
$$

has $F_{1}=F=M$ and

$$
F_{0}=\left(\begin{array}{cc}
0 & -T_{\beta(M)} \\
T_{\beta(F)} & 0
\end{array}\right)=J\left(\begin{array}{cc}
T_{\beta(F)} & 0 \\
0 & T_{\beta(F)}
\end{array}\right)=J T_{\beta(F)} \in J \mathrm{O}(n) .
$$

Now by definition $\Phi$ vanishes on $J \mathrm{O}(n)$, so $\Phi\left(F_{0}\right)=0$. We integrate

$$
\begin{aligned}
\int_{F_{s}} \chi_{S p(2 n)} & =\frac{1}{\pi} \int_{F_{s}} \operatorname{Tr}\left(\alpha_{s}^{t} \mathbf{d} \beta_{s}-\beta_{s}^{t} \mathbf{d} \alpha_{s}\right) \\
& =\frac{1}{\pi} \int_{F_{s}} \operatorname{Tr}\left(\cos \left(X_{s}\right) T_{\alpha(F)}^{t} T_{\beta(F)} \cos \left(X_{s}\right) \mathbf{d} X_{s}+\sin \left(X_{s}\right) T_{\beta(F)}^{t} T_{\alpha(F)} \sin \left(X_{s}\right) \mathbf{d} X_{s}\right) \\
& =\frac{1}{\pi} \int_{F_{s}} \operatorname{Tr}\left(T_{\alpha(F)}^{t} T_{\beta(F)} \mathbf{d} X_{s}\right) \\
& =\frac{1}{\pi} \operatorname{Tr}\left(T_{\alpha(F)}^{t} T_{\beta(F)}\left(X_{1}-X_{0}\right)\right) \\
& =\frac{1}{\pi} \operatorname{Tr}\left(T_{\alpha(F)}^{t} T_{\beta(F)}\left(X(F)-\frac{\pi}{2} \mathbf{1}_{n}\right)\right) \\
& =\Phi\left(F_{1}\right)=\Phi(F)
\end{aligned}
$$

by definition 13. of $\Phi$. Here we have used that $X_{s}, \mathbf{d} X_{s}, T_{\alpha(F)}^{t} T_{\beta(F)}$ commute, together with the identity $\cos ^{2}\left(X_{s}\right)+\sin ^{2}\left(X_{s}\right)=\mathbf{1}_{n}$. 
Since $F_{s}$ defined by 22 depends smoothly on $F$, we have a deformation retraction of $\mathrm{U}^{\times}(n)$ (and $\mathrm{Sp}^{\times}(2 n)$ ) on $J \mathrm{O}(n) \cong \mathrm{O}(n)$. Therefore any path $F(t) \in \mathrm{U}^{\times}(n)$ is homotopic relative end points to the catenation $a * T * b$ of two paths $a, b$ of type 22 and a path $T$ in $J \mathrm{O}(n)$. Since $\chi_{S p(2 n)}$ is the pull back of the closed form $\chi$ on $\mathrm{U}(1)$ it is closed as well and therefore line integrals over $\chi_{S p(2 n)}$ depend on the homotopy class relative end points only. For an arbitrary path $F$ in $\mathrm{U}^{\times}(n)$ we can therefore compute

$$
\int_{F} \chi_{S p(2 n)}=\int_{a * T * b} \chi_{S p(2 n)}=\int_{a} \chi_{S p(2 n)}+\int_{T} \chi_{S p(2 n)}+\int_{b} \chi_{S p(2 n)} .
$$

The integral over $T$ vanishes since $(J T)^{-1} \mathbf{d}(J T)=T^{-1} \mathbf{d} T$ has trace 0 . The integrals over $a$ and $b$ have been evaluated in $(23)$. Thus we get that

$$
\int_{F} \chi_{\operatorname{Sp}(2 n)}=\Phi(F(1))-\Phi(F(0))
$$

for any path $F(t)$ in $\mathrm{U}^{\times}(n)$ which proves the Lemma.

We now proceed to verify the axioms from [6] for the Maslov index for $\widetilde{\mu}$.

1. Homotopy: Two paths in $\operatorname{Sp}(2 n)$ are homotopic relative end points if and only if the respective values of $\widetilde{\mu}$ coincide.

Since $\chi_{\mathrm{Sp}(2 n)}=\left(\left(\operatorname{det}_{\mathbb{C}}^{2}\right) \circ F\right)^{*} \chi$ is closed we have that $\widetilde{\mu}$ is homotopy invariant relative end points. The map $\left(\operatorname{det}_{\mathbb{C}}^{2}\right) \circ F$ induces an injective map $\pi_{1}(\operatorname{Sp}(2 n)) \rightarrow \pi_{1}(\mathrm{U}(1))$ and the isomorphism $\pi_{1}(U(1)) \cong \mathbb{Z}$ is given by integrating the form $\chi$. For closed loops the end point terms (14) cancel and the claim follows.

2. Catenation: Let $M_{i}(t), i=0,1$ be paths in $\operatorname{Sp}(2 n)$ with $M_{0}(1)=M_{1}(0)$ and $M=M_{0} * M_{1}$ denote the path with $M(t)=M_{0}(2 t)$ for $t \leq 1 / 2$ and $M_{1}(2 t-1)$ for $t \geq 1 / 2$. Then $\widetilde{\mu}(M)=\widetilde{\mu}\left(M_{0}\right)+\widetilde{\mu}\left(M_{1}\right)$.

The contributions of the end points $M_{0}(1)$ and $M_{1}(0)$ cancel. The claim thus follows from the additivity of the integral.

3. Product: If $M(t)=\left(\begin{array}{cc}M_{1}(t) & 0 \\ 0 & M_{2}(t)\end{array}\right)$ with $M_{i}(t) \in \operatorname{Sp}\left(2 n_{i}\right), n_{1}+$ $n_{2}=n$, then $\widetilde{\mu}\left(M_{1}\right)+\widetilde{\mu}\left(M_{2}\right)=\widetilde{\mu}(M)$.

This follows from the analogous property of the trace.

4. Zero: Let $M(t)$ be a path such that $\operatorname{dim}\left(M(t) \Lambda_{0} \cap \Lambda_{0}\right)=k>0$ is constant for all $t$. Then $\widetilde{\mu}(M)=0$.

By lemma 19 we may replace $M(t)$ by $M(t) H(t)$ with $H(t) \in \mathrm{H}(n)$ without changing $\widetilde{\mu}$. We can thus achieve that that

$$
M(t)=\mathcal{F}(M(t))=\left(\begin{array}{cc}
\alpha(t) & -\beta(t) \\
\beta(t) & \alpha(t)
\end{array}\right) \in \mathrm{U}(n)
$$

is unitary. We also have $\operatorname{ker} \beta(t)=M(t) \Lambda_{0} \cap \Lambda_{0}$ which is of constant dimension $k$ by our assumption. Also $\alpha(t)$ maps $\operatorname{ker} \beta(t)$ isometrically into $\Lambda_{0}$. Therefore 
replacing $M(t)$ by $T_{1}(t) M(t) T_{2}(t)$ with suitable paths $T_{1}(t), T_{2}(t) \in \mathrm{O}(n)$, we may assume that $V=\operatorname{ker} \beta(t)$ is independent of $t$ and that $\alpha(t)$ is the identity on $V$. Again by Lemma 19 , we have $\widetilde{\mu}(M)=\widetilde{\mu}\left(T_{1} M T_{2}\right)$.

Let $W$ be the orthogonal complement of $V$ in $\Lambda_{0}$. We then can split

$$
\mathbb{R}^{2 n}=(V \oplus J V) \oplus(W \oplus J W)
$$

as the orthogonal sum of two symplectic subspaces. With respect to this splitting we now have

$$
M(t)=\left(\begin{array}{cc}
\mathbf{1}_{V \oplus J V} & 0 \\
0 & Q(t)
\end{array}\right) .
$$

The path $Q(t) \in \mathrm{Sp}^{\times}(W \oplus J W)=\mathrm{Sp}^{\times}(2(n-k))$ avoids the Maslov cycle. By Lemma 21. $\widetilde{\mu}(M)=\widetilde{\mu}(\mathbf{1})+\widetilde{\mu}(Q)=0$.

5. Normalization: Let $Y(t) \in \operatorname{Sym}(n)$ be a path of symmetric matrices and $M(t)=\left(\begin{array}{cc}\mathbf{1} & 0 \\ Y(t) & \mathbf{1}\end{array}\right)$ the corresponding path of symplectic shears. Then

$$
\widetilde{\mu}(M)=[\operatorname{sign}(Y(1))-\operatorname{sign}(Y(0))] / 2,
$$

where the signature $\operatorname{sign}(Y)$ is the number of positive eigenvalues of $Y$ minus the number of negative eigenvalues.

To see this, let $T(t) \in \mathrm{O}(n)$ be such that

$$
T(t)^{-1} Y(t) T(t)=\left(\begin{array}{ccc}
\lambda_{1}(t) & & \\
& \ddots & \\
& & \lambda_{n}(t)
\end{array}\right)
$$

is diagonal. Then

$$
\begin{array}{r}
T^{-1}(t) M(t) T(t)=\left(\begin{array}{cc}
T(t)^{-1} & 0 \\
0 & T(t)^{-1}
\end{array}\right)\left(\begin{array}{cc}
\mathbf{1} & 0 \\
Y(t) & \mathbf{1}
\end{array}\right)\left(\begin{array}{cc}
T(t) & 0 \\
0 & T(t)
\end{array}\right) \\
=\left(\begin{array}{cc}
\mathbf{1} & 0 \\
T(t)^{-1} Y(t) T(t) & \mathbf{1}
\end{array}\right) .
\end{array}
$$

By Lemma 19 and the product property,

$$
\widetilde{\mu}(M)=\widetilde{\mu}\left(T^{-1} M T\right)=\sum_{k=0}^{n} \widetilde{\mu}\left(\begin{array}{cc}
1 & 0 \\
\lambda_{k}(t) & 1
\end{array}\right) .
$$

It suffices therefore to verify the normalization property in the case $n=1$, $Y(t)=\lambda(t)$ and $M(t)=\left(\begin{array}{cc}1 & 0 \\ \lambda(t) & 1\end{array}\right)$. The integral in 14, i.e. the integral over the form (17), becomes

$$
\int_{M} \chi_{\operatorname{Sp}(2)}=\frac{-1}{2 \pi} \int_{M}-\mathbf{d} \lambda-\left(1+\lambda^{2}\right) \mathbf{d}\left(\left(1+\lambda^{2}\right)^{-1} \lambda\right)=\frac{[\arctan (\lambda(t))]_{t=0}^{t=1}}{\pi}
$$


For the end point term in 14 we compute

$$
F=\mathcal{F}\left(\begin{array}{cc}
1 & 0 \\
\lambda & 1
\end{array}\right)=\frac{1}{\sqrt{1+\lambda^{2}}}\left(\begin{array}{cc}
1 & -\lambda \\
\lambda & 1
\end{array}\right)
$$

which gives

$$
X=\arcsin \left(\frac{|\lambda|}{\sqrt{1+\lambda^{2}}}\right)=\arctan (|\lambda|) \quad, \quad T_{\beta}=\operatorname{sign}(\lambda) \quad, \quad T_{\alpha}=1
$$

in the notation of (11) and (12). From 13 we get

$$
\Phi(M)=\left\{\begin{array}{clc}
\frac{1}{\pi} \arctan (\lambda)-\frac{1}{2} & \text { if } \quad \lambda>0 \\
0 & \text { if } \quad \lambda=0 \\
\frac{1}{\pi} \arctan (\lambda)+\frac{1}{2} & \text { if } \quad \lambda<0
\end{array} .\right.
$$

This yields $\widetilde{\mu}(M)=[\operatorname{sign}(\lambda(1))-\operatorname{sign}(\lambda(0))] / 2$ as required.

\section{References}

[1] V. I. Arnol'd On a characteristic class entering into conditions of quantization Functional Analysis 1 (1967), 1-8.

[2] M. C. Gutzwiller Chaos in classical and quantum mechanics Springer, New York, 1990

[3] M. Brack, M. Pletyukhov On the canonically invariant calculation of Maslov indices J. Phys. A 36 (2003), no. 36, 9449-9469.

[4] M. Brack, R. K. Bhaduri Semiclassical physics Frontiers in Physics, 96. Westview Press, Boulder, CO, 2003

[5] D. McDuff, D. Salamon Introduction to symplectic topology Oxford Mathematical Monographs. The Clarendon Press, Oxford University Press, New York, 1998

[6] J. Robbin, D. Salamon The Maslov Index for Paths Topology 32 no 4 (1993), 827-844 